\title{
Aceitação sensorial e determinação da composição centesimal de biscoitos adicionados de farinha de alfarroba (Ceratonia siliqua $L$.)
}

\section{Sensory acceptance and determination of the centesimal composition of biscuits added with carob flour (Ceratonia siliqua $L$.)}

\author{
Mauricio Rigo ${ }^{1 *}$ \\ Raniere Mazilde Vidal Bezerra² \\ Sabrina Ferreira Bezerra ${ }^{3}$ \\ Ângela Moraes Teixeira ${ }^{4}$
}

\section{Resumo}

O objetivo deste trabalho foi desenvolver quatro formulações de biscoitos, sendo uma sem adição de farinha de alfarroba (FP) e as demais acrescidas de 20\% (F1), 30\% (F2) e 40\% (F3) de farinha de alfarrob,a em substituição parcial à farinha de trigo e avaliar sua aceitação sensorial e composição centesimal. $\mathrm{Na}$ análise sensorial, foram avaliados os atributos: aceitação global, aparência, aroma, sabor, textura e cor, por meio de escala hedônica de nove pontos. Todas as formulações de biscoitos receberam notas superiores a seis, sendo que as formulações com farinha de alfarroba apresentaram as melhores notas. As formulações com farinha de alfarroba apresentaram maior aceitação na cor $(p<0,05)$, do que na FP. Para todos os outros atributos sensoriais investigados não houve diferença estatística significativa $(\mathrm{p}>0,05)$ entre as formulações. A F1 apresentou maiores teores de fibra bruta e cinzas do que a FP. Pode-se concluir que a adição de até $40 \%$ farinha de alfarroba em biscoitos resulta em produtos boa aceitação sensorial.

Palavras-chave: panificação, sobremesa, aceitação sensorial.

\section{Abstract}

The objective of this work was to develop four biscuit formulations, one without carob flour (PF) and the other 20\% (F1), 30\% (F2) and 40\% (F3) of carob flour, in substitution wheat flour, and to evaluate its sensorial acceptance and centesimal composition. In the sensorial analysis were evaluated the attributes: global acceptance, appearance, aroma, flavor, texture and color, through a hedonic scale of nine points. All the biscuit formulations received marks superior to six, the formulations with carob flour presented the best grades. The formulations with carob flour showed greater color acceptance ( $\mathrm{p}$ $<0.05$ ), than in FP. For all other sensory attributes investigated there was no significant statistical difference $(\mathrm{p}>0.05)$ between the formulations. The F1 presented higher levels

I Dr.; Engenheiro de Alimentos; Professor da Universidade Estadual do Centro Oeste, Unicentro; Rua SimeãoVarela de Sá, 03,Vila Carli, CEP: 85040-080, Guarapuva-PR, Brasil; E-mail: mrigo@unicentro.br; (*) Autor para correspondência;

2 Dr.; Engenheiro de Alimentos; Professor da Universidade Estadual do Centro-Oeste, UNICENTRO; Endereço: Rua Simeão Varella de Sá, 03,Vila Carli, CEP. 85040-080, Guarapuava, Paraná, Brasil; E-mail: raniere@unicentro.br;

3 Graduanda em Engenharia de Alimentos; Universidade Estadual do Centro-Oeste, UNICENTRO; Endereço: Rua Simeão Varella de Sá, 03,Vila Carli, CEP. 85040-080, Guarapuava, Paraná, Brasil; E-mail: sbezera I @hotmail.com;

4 Dra.; Engenheira de Alimentos; Professora da Universidade Estadual do Centro-Oeste, UNICENTRO; Endereço: Rua Simeão Varella de Sá, 03,Vila Carli, CEP. 85040-080, Guarapuava, Paraná, Brasil; E-mail: amteixeira@yahoo.com.br.

\begin{tabular}{llllll}
\hline Ambiência & Guarapuava (PR) & v.l5 n.2 & p. 333 - 342 & Maio/Ago 2019 & ISSN I808 - 025I
\end{tabular}


of moisture and ash than FP. It can be concluded that the addition of up to $40 \%$ carob flour in biscuits results in good sensory acceptance.

Keywords: bakery, dessert, sensory acceptance.

\section{Introdução}

Os principais ingredientes para fazer biscoitos são farinhas, açúcares e gorduras. A farinha de trigo composta por proteínas, incluindo gliadina e glutenina que, na presença de água se combinam para formar o glúten. A formação do glúten, sua força e elasticidade são determinadas em grande parte pelo teor de proteína da farinha, ingredientes e processo tecnológico de mistura e moldagem dos biscoitos (DAVIDSON, 2018).

O amido é o principal componente da farinha de trigo. Representa quase todo o conteúdo de carboidratos e cerca de $80 \%$ do conteúdo energético total da farinha de trigo. Açúcar dá doçura, mas também é importante no desenvolvimento da textura do biscoito (DAVIDSON, 2018).

As gorduras são ingredientes de importância vital para alcançar a textura, a sensação na boca e a mordida do biscoito. As gorduras são produzidas a partir de óleos crus de boa qualidade por um processo de refino, branqueamento e desodorização. Eles são produzidos principalmente a partir de óleos vegetais, mas pode conter óleos de peixe hidrogenados (DAVIDSON, 2018).

Segundo dados da Associação Brasileira das Indústrias de Biscoitos, Massas Alimentícias e Pães, no ano de 2017, foram vendidos, nos dez países que mais consomem biscoito no mundo, 14.389,8 mil toneladas de biscoitos no valor de 68,5 bilhões de dólares. No Brasil, foram vendidos 1.818 mil toneladas no valor de 24 bilhões de reais, sendo o consumo per capita de 8,76 kg/ano em 2017 (ABIMAP, 2019).

A inovação de produtos com qualidade sensorial e nutricional que apresentem benefícios associados à saúde tem sido a chave para a sobrevivência das indústrias alimentícias no Brasil, as quais vêm desenvolvendo novos produtos visando atender a demanda dos consumidores por alimentos que promovam a saúde, bem-estar e a redução de riscos de doenças. $\mathrm{Na}$ área de panificação, a utilização de farinhas mistas para elaboração de novos produtos, com utilização de componentes que incrementem os teores de fibras e/ou proteínas, ou possua algum componente funcional no produto final tem sido alvo de investigação (RIGO, et al., 2017; RIGO, et al., 2018).

A alfarroba é uma vagem comestível semelhante ao feijão com coloração marrom escuro e sabor adocicado, nativa dos países do mediterrâneo (CREDIDIO, 2006). Após a remoção das sementes a alfarroba pode ser processada para obtenção da farinha ou pó destinada à alimentação humana ou utilizada nas indústrias de alimentos e farmacêuticas (BARRACOSA, et al., 2009).

A alfarroba oferece diversas atividades antioxidantes, anticâncer e antidiabéticos, devido à presença de uma grande quantidade de pinitol e antibacterianos. Rica em açúcares, proteínas e minerais, a alfarroba tem uma vasta gama de aplicações industriais, como substitutos do cacau, substitutos dos açúcares, livres do glúten, tornando-os alimentos derivados de cereais para pessoas celíacas (BOUBLENZA, et al., 2019).

A alfarroba é usada em muitos países árabes para fazer bebida popular que é consumida principalmente no mês do Ramadã. A alfarroba também é usada na preparação de produtos tradicionais da confeitaria árabe, e o pó ou farinha de alfarroba é comercializado em países ocidentais em lojas de alimentos saudáveis como substituto ou extensor do cacau (YOUSIF \& ALGHZAWI, 2000). 
A farinha de alfarroba possui cor, sabor e aroma semelhantes ao do cacau e pó, porém com o benefício de não possuir os compostos estimulantes encontrados no cacau (cafeína e teobromina) e que são considerados como fatores antinutricionais por serem responsáveis por efeitos fisiológicos adversos (GROSSO \& BRACKEN, 2005). A farinha de alfarroba possui preço menor do que o cacau em pó, portanto pode ser utilizada como um substituto de cacau na formulação de biscoitos, massas, sobremesas, bebidas lácteas e achocolatados, com o objetivo de redução de custo e acrescentar alguma característica nova ao produto final.

Diversos pesquisadores têm estudado formulações de biscoitos com farinhas alternativas, investigando os aspectos tecnológicos, sensoriais e nutricionais dos biscoitos. Čukelj e colaboradores (2017) investigaram as propriedades nutricionais (lignanas, ômega-3, ácidos fenólicos, atividade antioxidante), físicas (textura, espalhamento) e sensoriais de biscoitos feitos de trigo integral, centeio, aveia e cevada com adição de linhaça moída (10\% sobre base de farinha), bem como a estabilidade oxidativa durante o armazenamento. Feddern e colaboradores (2011) avaliaram biscoitos elaborados com até $30 \%$ de farelo de arroz e biscoitos elaborados com até $45 \%$ de farelo de trigo. Os resultados apontam que os atributos sensoriais do biscoito com $20 \%$ de farelo de arroz foram iguais ou melhores que o controle, com exceção da mastigabilidade. Os biscoitos elaborados com farelo de trigo não diferiram entre si em quatro dos sete atributos sensoriais estudados. Os autores concluíram que os farelos estudados podem ser adicionados em biscoitos ( $15 \%$ de farelo de trigo e $20 \%$ de farelo de arroz) sem prejuízos às características físicas e sensoriais destes, com mercado consumidor potencial.

Sabatini e colaboradores (2011) estudaram a composição química da farinha de alfarroba e desenvolveram uma formulação de sorvete com farinha de alfarroba, cujos resultados dos testes de aceitação e intenção de compra foram bons, com valores de índice de aceitabilidade para todos os atributos investigados superiores a $80 \%$ e porcentagem de aprovação do teste de intenção de compra de $93 \%$, e concluíram que o sorvete de alfarroba desenvolvido ofereceu boas perspectivas de consumo e, portanto, poderia ser produzido em escala comercial. A farinha de alfarroba também apresentou alto teor de fibra bruta 5,68 \% e baixo teor de lipídeos 0,48 \%.

Atualmente os hábitos alimentares da população trazem baixa ingestão de fibras, vitaminas e minerais devido ao baixo consumo de vegetais frescos. Uma maneira possível de aumentar o consumo desses nutrientes é o desenvolvimento de novos produtos ricos nesses nutrientes. Uma alternativa que se apresenta é o emprego de novos ingredientes às formulações dos produtos para enriquecer o valor nutricional de alimentos tradicionais (VORAGEN, 1998).

Medeiros e Lannes (2009) reportaram o uso de farinha de alfarroba como substituto de cacau em pó na formulação e bebidas lácteas, biscoitos, bolos, sorvetes e achocolatados, a qual acrescentou uma característica nova ao produto final.

Freitas Júnior e colaboradores (2017) investigaram o uso de farinha de alfarroba em substituição ao chocolate em cookies, doces tipo brigadeiro, iogurte e leite não fermentado. Através da avaliação sensorial dos produtos elaborados concluíram que os mesmos tiveram boa aceitação sensorial e apresentaram-se mais saudáveis e com alegação funcional.

Barroso e colaboradores (2015) investigaram a viabilidade da substituição do cacau por alfarroba em pó no desenvolvimento de biscoitos recheados e avaliaram suas características químicas e sensoriais. Três formulações foram testadas: biscoitos de alfarroba, de alfarroba com proteína de soja, e de cacau. Os resultados das análises químicas para umidade, cinzas, proteínas 
e lipídeos apresentaram-se semelhantes para todas as amostras, sendo que o biscoito de cacau teve maior teor de fibra bruta. Os resultados do teste de aceitação sensorial para os atributos aparência, textura, sabor e aceitação global não apresentaram diferença estatística significativa entre as formulações ( $p>0,05)$. Os autores concluíram que os biscoitos de alfarroba e de alfarroba com proteína de soja, mantiveram as características sensoriais e nutricionais quando comparados com biscoito de cacau.

A procura por alimentos mais saudáveis por parte dos consumidores tem impulsionado o desenvolvimento de produtos similares aos convencionais que contenham menor teor de gordura e maior teor de fibras, sem alterar as características sensoriais dos produtos. Portanto o biscoito com substituição parcial da farinha de trigo pela de alfarroba pode ser uma opção de produto a ser desenvolvido e submetido à apreciação dos consumidores.

Nesse escopo, este trabalho teve como objetivos a elaboração de formulações de biscoitos com farinha de alfarroba, em substituição parcial a farinha de trigo, e a avaliação de suas características físico-químicas e sensoriais.

\section{Material e Métodos}

\subsection{Local de pesquisa}

Este trabalho foi desenvolvido no Departamento de Engenharia de Alimentos da Universidade Estadual do Centro-Oeste, nos laboratórios de Análise de Alimentos, Processos na Indústria de Alimentos e Analise Sensorial.

\subsection{Obtenção da matéria prima}

Os ingredientes foram comprados em supermercados de Guarapuava, PR, sendo que a farinha de alfarroba foi adquirida em loja de produtos naturais.

\section{3. Preparo dos biscoitos}

As formulações de biscoitos foram desenvolvidas a partir de testes preliminares, resultando nas composições apresentadas na Tabela 1. Foram elaboradas as formulações de biscoito padrão (FP) sem farinha de alfarroba e as formulações F1, F2 e F3 contendo 20\%, 30\% e 40\% de farinha de alfarroba, em substituição parcial a farinha de trigo, respectivamente.

\section{Tabela 1. Formulações dos biscoitos com diferentes proporções de farinha de alfarroba.}

\begin{tabular}{|c|c|c|c|c|}
\hline & \multicolumn{4}{|c|}{ Formulações } \\
\cline { 1 - 1 } Ingredientes & FP (\%) & F1 (\%) & F2 (\%) & F3 (\%) \\
\cline { 1 - 1 } Farinha de trigo & 26,3 & 21,4 & 18,4 & 15,8 \\
\cline { 1 - 1 } Aveia & 14,3 & 14,3 & 14,3 & 14,3 \\
\cline { 1 - 1 } Óleo de coco & 15,1 & 15,1 & 15,1 & 15,1 \\
\cline { 1 - 1 } Açúcar mascavo & 12,8 & 12,8 & 12,8 & 12,8 \\
\cline { 1 - 1 } Ovos & 16,7 & 16,7 & 16,7 & 16,7 \\
\cline { 1 - 1 } Fermento em pó & 4,8 & 4,8 & 4,8 & 4,8 \\
\cline { 1 - 1 } Canela & 0,3 & 0,3 & 0,3 & 0,3 \\
Farinha de Alfarroba & 0 & 5,3 & 7,9 & 10,5 \\
Mix de cereais & 9,6 & 9,6 & 9,6 & 9,6 \\
\hline
\end{tabular}

Nota: FP_ Formulação padrão sem adição de farinha de alfarroba; F1 - Formulação com 20\% de farinha de alfarroba; F2 - Formulação com 30\% de farinha de alfarroba e F3 - Formulação com 40\% de farinha de alfarroba. 
Para elaboração da massa, misturaram-se manualmente os ingredientes secos, em seguida foram adicionados o ovo e óleo de coco derretido. A massa foi homogeneizada por 5 minutos. A seguir a massa foi aberta e cortada em círculos de $40 \mathrm{~mm}$ de diâmetro e espessura de $6 \mathrm{~mm}$, os quais foram levados ao forno a gás (Marca Venâncio, Brasil) a $160{ }^{\circ} \mathrm{C}$ durante aproximadamente 25 minutos.

\subsection{Avaliação Sensorial}

Considerando-se o interesse e disponibilidade, 60 provadores não treinados da comunidade acadêmica da Universidade Estadual do Centro-Oeste participaram do teste de aceitação, todos com idade entre 18 e 50 anos. Todos os participantes assinaram o Termo de Consentimento Livre e Esclarecido antes da sessão sensorial.

Todas as formulações desenvolvidas foram avaliadas quanto à aparência, textura, aroma, sabor, cor e aceitação global, utilizando-se escala hedônica estruturada de nove pontos, cujos extremos correspondem a gostei muitíssimo (9) e desgostei muitíssimo (1). As amostras foram oferecidas em copos plásticos, codificadas com números de três dígitos aleatórios, acompanhadas de um copo de água para realização do branco entre as amostras. As formulações foram oferecidas aos julgadores de forma monódica sequencial (DUTCOSKY, 2007).

Os resultados dos testes sensoriais foram tratados por análise de variância (ANOVA) e o Teste de Tukey foi usado para verificar diferenças estatísticas entre as amostras, ambos ao nível de 5\% de significância. O programa empregado foi o Statgraphics plus, versão 5.6.

Este trabalho foi aprovado pelo Comitê de Ética em Pesquisa da UNICENTRO em 02 de agosto de 2016, protocolo no 57739116.0.0000.0106.

Entretanto, como critérios de exclusão foram considerados os seguintes fatores: possuir alergia a algum ingrediente utilizado na elaboração dos produtos, possuir idade maior ou menor do que a faixa de interesse do estudo, não ser aluno ou funcionário do campus em questão e as pessoas que não assinaram o Termo de Consentimento Livre e Esclarecido (TCLE).

\subsection{Análises Físico-Químicas}

As análises físico-químicas foram feitas na farinha de alfarroba e nas formulações de biscoitos. Todas as análises foram feitas em triplicata.

O conteúdo de umidade foi determinado através do método gravimétrico, baseando-se na perda de peso do material submetido ao aquecimento em estufa (Marca Odontobras, Brasil) a $105^{\circ} \mathrm{C}$ até peso constante.

O teor de proteínas foi determinado através da avaliação do nitrogênio total da amostra, pelo método Kjeldahl (AACC,1995). Utilizou-se o fator de conversão de nitrogênio para proteína de 6,25.

O teor de lipídeos foi determinado pelo método de extração a frio (BLIGH \&DYER,1959).

O teor de cinzas foi determinado por incineração do material em mufla (Marca Quimis, Brasil) a $550{ }^{\circ} \mathrm{C}$ até peso constante (AOAC, 2000).

A quantificação de fibra bruta foi determinada pelo método de extração em ebulição, após uma digestão ácida e outra alcalina. A primeira extração foi com solução de $\mathrm{H}_{2} \mathrm{SO}_{4}(1,25 \% \mathrm{p} / \mathrm{v})$ por 30 minutos, seguida de filtração e lavagem. A segunda extração foi com $\mathrm{NaOH}(1,25 \%$ p/v) por mais 30 minutos, seguida por filtração e lavagem, após secagem a $100^{\circ} \mathrm{C}$ até peso constante (BRASIL, 1991).

A quantidade de carboidrato foi determinada por diferença, subtraindo-se de 100 os teores em porcentagem de umidade, proteína, cinza e lipídeo. $\mathrm{O}$ valor dos carboidratos inclui as fibras totais. 


\section{Resultados e Discussão}

A composição centesimal da farinha de alfarroba e da farinha de trigo, usadas na produção dos biscoitos neste trabalho, estão apresentadas na Tabela 2. Segundo a Tabela Brasileira de Composição de Alimentos - TACO (2011), a farinha de trigo apresentou teores de fibras, carboidratos e cinzas menores do que os reportados para a farinha de alfarroba dete estudo, e o valor do teor de fibras obtido para a farinha de alfarroba de 6,79 \% foi três vezes superior ao valor de fibras na farinha de trigo de 2,3 \%. A utilização da farinha de alfarroba como substituto parcial da farinha de trigo torna-se promissor para grupos de consumidores com carências nutricionais de fibras.

\section{Tabela 2: Composição centesimal da farinha de alfarroba e da farinha de trigo.}

\begin{tabular}{|c|c|c|}
\hline Parâmetros (\%) & $\begin{array}{c}\text { Farinha de alfarroba } \\
*\end{array}$ & $\begin{array}{c}\text { Farinha de trigo } \\
\text { (TACO 2011) }\end{array}$ \\
\hline Umidade & $10,6 \pm 0,05$ & 13 \\
\hline Cinzas & $2,98 \pm 0,03$ & 0,8 \\
\hline Proteínas & $5,15 \pm 0,35$ & 9,8 \\
\hline Lipídeos & $0,62 \pm 0,21$ & 1,4 \\
\hline Fibra Bruta & $6,79 \pm 0,88$ & 2,3 \\
\hline Carboidratos & $80,65 \pm 0,41$ & 75,1 \\
\hline
\end{tabular}

Nota: * Valores relativos à média \pm desvio padrão.

Sabatini e colaboradores (2011) estudaram a elaboração de sorvete com pó de alfarroba e apresentaram os resultados das análises físico-químicas para alfarroba em pó de: umidade 8,76 $\%$, cinzas 3,67 \%, lipídeos 0,48 \%, fibra bruta 5,68 \%, proteínas 4,79 \% e carboidratos 76,6 \%, portanto, esses valores corroboram os resultados das análises físico-químicas para farinha de alfarroba obtidas neste trabalho (Tabela 2).

As notas médias obtidas nos testes de aceitação das formulações de biscoitos avaliando os atributos sensoriais: aceitação global, aparência, aroma, sabor, textura, e cor dos tratamentos FP, F1, F2 e F3 estão apresentadas na Tabela 3.

\section{Tabela 3: Notas dos testes de aceitação dos atributos sensoriais aceitação global, aparência, aroma, sabor, textura, e cor das formulações de biscoitos.}

\begin{tabular}{|c|c|c|c|c|}
\hline \multirow{2}{*}{$\begin{array}{c}\text { Atributos } \\
\text { Sensoriais }\end{array}$} & \multicolumn{4}{|c|}{ Formulações } \\
\cline { 2 - 5 } & FP (\%) & F1 (\%) & F2 (\%) & F3 (\%) \\
\hline Aceitação global & $7,12 \pm 1,41^{\mathrm{a}}$ & $7,52 \pm 1,15^{\mathrm{a}}$ & $7,38 \pm 1,28^{\mathrm{a}}$ & $7,04 \pm 1,6^{\mathrm{a}}$ \\
\hline Aparência & $6,90 \pm 1,57^{\mathrm{a}}$ & $7,48 \pm 1,3^{\mathrm{a}}$ & $7,54 \pm 1,09^{\mathrm{a}}$ & $7,2 \pm 1,56^{\mathrm{a}}$ \\
\hline Aroma & $7,48 \pm 1,31^{\mathrm{a}}$ & $7,46 \pm 1,37^{\mathrm{a}}$ & $7,18 \pm 1,32^{\mathrm{a}}$ & $7,04 \pm 1,46^{\mathrm{a}}$ \\
\hline Sabor & $6,88 \pm 1,29^{\mathrm{a}}$ & $7,56 \pm 1,39^{\mathrm{a}}$ & $7,32 \pm 1,3^{5} \mathrm{a}$ & $6,9 \pm 1,68^{\mathrm{a}}$ \\
Textura & $6,86 \pm 1,39^{\mathrm{a}}$ & $7,24 \pm 1,42^{\mathrm{a}}$ & $7,1 \pm 1,39^{\mathrm{a}}$ & $7,02 \pm 1,52^{\mathrm{a}}$ \\
Cor & $6,84 \pm 1,35^{\mathrm{a}}$ & $7,56 \pm 1,23^{\mathrm{b}}$ & $7,58 \pm 1,25^{\mathrm{b}}$ & $7,56 \pm 1,28^{\mathrm{b}}$ \\
\hline
\end{tabular}

Nota: FP- Formulação padrão sem adição de farinha de alfarroba; F1 - Formulação com 20\% de farinha de alfarroba; F2 - Formulação com 30\% de farinha de alfarroba e F3 - Formulação com 40\% de farinha de alfarroba. Médias com letras iguais na mesma linha indicam não haver diferença estatística significativa entre os resultados $(p>0,05)$, pelo teste de Tukey. 
Para a formulação padrão, o resultado do teste de aceitação para o atributo cor diferiu com $5 \%$ de significância das formulações com adição de farinha de alfarroba, sendo que as notas maiores foram para as formulações com a farinha de alfarroba, com médias em torno de 7,56 que correspondem a "gostei moderadamente" a "gostei muito". Para todos os outros atributos sensoriais investigados não houve diferença estatística significativa $(p>0,05)$ entre as formulações (Tabela 3 ).

De modo geral as notas do teste de aceitação ficaram entre a escala hedônica de "gostei moderadamente" a "gostei muito", sendo que as formulações de biscoito contendo farinha de alfarroba apresentaram as melhores notas na avaliação sensorial, destacando-se a formulação F1 com 20\% de farinha de alfarroba. Esses resultados apresentaram notas maiores do que os reportados por Barroso e colaboradores (2015), que estudaram a viabilidade da substituição do cacau por alfarroba em pó no desenvolvimento de biscoitos recheados e avaliaram suas características sensoriais. Três formulações foram testadas: biscoitos com alfarroba, alfarroba e proteína de soja, e cacau. Os resultados obtidos para os atributos aparência, textura, sabor e aceitação global não apresentaram diferença estatística significativa entre as formulações ( $p>0,05)$, as notas obtidas para os biscoitos com adição de farinha de alfarroba ficaram entre 6,6 e 6,9 ("gostei ligeiramente" a "gostei moderadamente"). A cor apresentou maior aceitação em biscoitos de cacau $(p<0,05)$, comparado às outras duas formulações. No entanto, a intenção de compra foi semelhante para todas as formulações e não apresentaram diferença significativa entre os resultados a 5 \% de confiança.

Em relação à aparência, todas as formulações de biscoito contendo farinha de alfarroba alcançaram médias entre 7 e 8 ("gostei moderadamente" a "gostei muito") mostrando uma elevada aceitação dos produtos. Para os provadores não houve prevalência entre os produtos quanto à aparência, pois não apresentaram diferença significativa $(p>0,05)$ entre os produtos com farinha de alfarroba. Ressalta-se que a primeira impressão que se tem de um alimento é geralmente visual, sendo que esse atributo é um dos aspectos considerados fundamentais na qualidade e aceitação dos alimentos.

A farinha de alfarroba possui aroma, sabor e cor parecidos com o cacau em pó, que em geral, é um produto apreciado pelos consumidores. Portanto é possível inferir que o fato de a farinha de alfarroba ter atributos sensoriais parecidos com o do cacau em pó contribuiu para a melhora da aceitação sensorial das formulações de biscoitos com farinha de alfarroba.

As altas notas do teste de aceitação para todos os atributos avaliados indicam que os biscoitos com adição de farinha de alfarroba, desenvolvidos neste trabalho, possuem potencial de consumo promissor no mercado, sendo plausível a utilização da farinha de alfarroba em substituição à farinha de trigo para melhoramento da aceitação sensorial de biscoitos. Ressalta-se que Yousif \& Alghzawi, (2000) estudaram o processamento de farinha de alfarroba e concluíram que ela de alfarroba é um alimento saudável natural que pode ser usado eficientemente como substituto ou extensor cacau em pó em muitos alimentos.

A formulação de biscoito com $20 \%$ de farinha de alfarroba apresentou as maiores notas no teste de aceitação sensorial para todos os atributos avaliados (7,24 a 7,56). Portanto, nessa formulação e na formulação padrão foram realizadas as análises físico-químicas e os resultados são apresentados na Tabela 4.

Os resultados da análise físico-química nas amostras de biscoitos mostram que o teor de fibra bruta, cinzas e lipídeos da formulação com $20 \%$ de alfarroba foram maiores do que os encontrados na amostra padrão. Apenas o teor de cinzas para as formulações FP e F1 apresentou diferença estatística significativa entre os resultados ao nível de $95 \%$ de significância. Como a farinha de alfarroba foi utilizada em substituição parcial da farinha de trigo na formulação F1, era esperado um acréscimo no teor de fibra bruta e cinzas nessa 


\section{Tabela 4. Características físico-químicas das formulações de biscoito padrão e com $20 \%$ de farinha de alfarroba, em base seca.}

\begin{tabular}{|c|c|c|}
\hline Parâmetros & FP (\%) & F1 (\%) \\
\hline Cinzas & $1,65 \pm 0,05^{\mathrm{a}}$ & $1,87 \pm 0,02^{\mathrm{b}}$ \\
\hline Lipídeos & $20,18 \pm 0,19^{\mathrm{a}}$ & $20,45 \pm 0,31^{\mathrm{a}}$ \\
\hline Fibra bruta & $3,16 \pm 0,66^{\mathrm{a}}$ & $3,70 \pm 0,87^{\mathrm{a}}$ \\
\hline Proteínas & $10,32 \pm 0,74^{\mathrm{a}}$ & $9,85 \pm 0,89^{\mathrm{a}}$ \\
\hline Carboidratos & $67,85 \pm 0,77^{\mathrm{a}}$ & $67,83 \pm 0,95^{\mathrm{a}}$ \\
\hline
\end{tabular}

Nota: FP- Formulação padrão sem adição de farinha de alfarroba; F1 - Formulação com $20 \%$ de farinha de alfarroba. Médias com letras iguais na mesma linha indicam não haver diferença estatística significativa entre os resultados ( $p>0,05)$, pelo teste de Tukey.

formulação em comparação à formulação padrão, pois a farinha de alfarroba possui teores de fibras e cinzas maiores do que os da farinha de trigo.

Os valores obtidos para os componentes lipídeos, fibra bruta, carboidratos e proteínas para as formulações FP e F1 não apresentaram diferença estatística significativa entre os resultados ( $>$ >0,05).

Barroso e colaboradores (2015) elaboraram biscoito de alfarroba e reportaram os seguintes resultados para a análise físico-química: cinzas 1,55\%, proteína 9,65\%, fibra bruta 1,3\%, lipídeos $12,15 \%$ e carboidratos $75,35 \%$. Os teores de cinzas e proteínas são da mesma ordem de grandeza dos apresentados neste trabalho (Tabela 4), para biscoito com $20 \%$ de farinha de alfarroba.

\section{Conclusões}

O desenvolvimento dos produtos permitiu comprovar que a adição de até $40 \%$ farinha de alfarroba em biscoitos, em substituição parcial da farinha de trigo, foi bem-aceita pelos julgadores para todos os atributos avaliados, obtendo-se aceitação sensorial melhor do que a do produto padrão sem a farinha de alfarroba. As três formulações de biscoitos com farinha de alfarroba não diferiram entre si para todos os atributos investigados a $(p>0,05)$. Portanto, as formulações elaboradas com farinha de alfarroba podem ser oferecidas aos consumidores com boas expectativas de aceitação no mercado.

As características físico-químicas dos biscoitos com $20 \%$ de farinha de alfarroba assemelharam-se aos dos respectivos biscoitos padrão. A análise físico-química do produto adicionado de $20 \%$ de farinha de alfarroba apontou um leve aumento nos teores de cinzas e fibras, e redução nos teores de proteínas e carboidratos, em comparação ao produto padrão.

Os resultados deste trabalho contribuem no desenvolvimento de produtos para uma nova demanda de consumidores que buscam uma alimentação com atrativos mais saudáveis e com qualidade sensorial.

\section{Referências}

AACC - AMERICAN ASSOCIATION OF CEREAL CHEMISTS. Approved methods. 9. ed. Saint Paul, 1995. 
ABIMAP. Associação Brasileira das Indústrias de Biscoitos, Massas Alimentícisas e Pães \& Bolos Industrializados. Estatísticas biscoitos. Disponível em: < https://www. abimapi.com.br/estatistica-biscoito.php>. Acesso em: 18 de abril de 2019.

AOAC - Analysis of association of official analytical chemists - AOAC. Official Methods of Analysis of Association of Official Analytical Chemists. 13 ed. Washington, DC, 2000.

BARRACOSA, P.; CAETANO, I.; BATISTA, M. T. Avaliação do rendimento agroindustrial dos frutos e sementes de cultivares da alfarrobeira (Ceratonia siliqua L.) no Algarve. Millenium, n. 37, novembro 2009.

BARROSO, L. S.; OLIVEIRA, V.R.; GARCIA, A. V.; DONEDA, D.; OURIQUES, L.A.; VIEIRA, M.M. Physicochemical and Sensory Evaluation of Sandwich Cookies Made with Carob Powder. Advance Journal of Food Science and Technology, v.9, n. 4, p. 290-295, 2015.

BLIGH, E.G.; DYER, W.J. A rapid method of total lipid extraction and purification. Canadian Journal Biochemistry and Physiology, Ottawa, v.37, n.8, p.911-917, 1959.

BOUBLENZA, I.; BOUBLENZA, I.; BOUBLENZA, A.; MADJI, S.; TIXIER, F. A. S.; CHEMAT, F. (2019) Carob as Source for Sustainable Ingredients and Products. In: Li Y., Chemat F. (eds) Plant Based "Green Chemistry 2.0". Green Chemistry and Sustainable Technology. Springer, Singapore

BRASIL. Portaria No 108, de 04 de setembro de 1991. Normais gerais de amostragem par análise de rotina. Método número 11 - Fibra Bruta. Diário Oficial da República Federativa do Brasil, Brasília, DF, p. 19813, 17 set. 1991.

ČUKELJ, N; NOVOTNI, D., SARAJLIJA, H.DRAKULA, S., VOUČKO, B., ĆURIĆ, D. Flaxseed and multigrain mixtures in the development of functional biscuits, LWT Food Science and Technology, Volume 86, p 85-92, 2017.

CREDIDIO, E. Alimentos Funcionais na Nutrologia médica, São Paulo: Ottoni 2006.

DAVIDSON, I. Ingredients for Biscuits: An Introduction. In: Biscuit, Cookie and Cracker Production (Second Edition). Academic Press, 2018. Chapter 16 p 165-172.

DUTCOSKY, S. D. Análise sensorial de alimentos. 2. Ed. Curitiba: Champagnat, 2007. $123 p$.

FEDDERN, V.; DURANTE, V. V. O.; MIRANDA, M. Z.; MELLADO, M. M. S. Avaliação física e sensorial de biscoitos tipo cookie adicionados de farelo de trigo e arroz. Brazilian Journal Food Technology., Campinas, v. 14, n. 4, p. 267-274, out./dez. 2011. 
FREITAS JÚNIOR, E, M; LEMOS, R. E. S.; CARVALHO, C. M. S.; SILVA, M. M. S.; ALVES V. R.; COSTA, Z. R. T.; NETO, L. G. M.; SOARES, D. J. Elaboração de produtos alimentícios com farinha de alfarroba (Ceratonia siliqua L.). Brazilian Journal of Food Research, Campo Mourão, v. 8, n.1, p. 80-90, jan./mar. 2017.

GROSSO, L. M.; BRACKEN, M. B. Caffeine metabolism, genetics, and perinatal outcomes: a review of exposure assessment considerations during pregnancy. Annals Epidemiology, v. 15, n. 6, p. 460-466, 2005.

MEDEIROS, M. L.; LANNES, S. C. S. Avaliação química de substitutos de cacau e estudo sensorial de achocolatados formulados. Ciência e Tecnologia de Alimentos, v. 29 , n. 2, p. 247-253, 2009.

RIGO,M.; BEZERRA,J.R.M.V.; RODRIGUES, D.D.; TEIXEIRA, A. M.nAvaliação físico-química e sensorial de biscoitos tipo cookie adicionados de farinha de bagaço de malte como fonte de fibra. Ambiência. v. 13, n. 1, p. 47 - 57, 2017.

RIGO, M.; TEIXEIRA, A. M.; CARLI, L. F.; BEZERRA, J. R. M. V. Avaliação físicoquímica e sensorial de pães com diferentes proporções de farinha de bagaço de cana-deaçúcar como fonte de fibra. Ambiência. v.14, n.3, p. 449 - 460, 2018.

SABATINI, D. R.; SILVA, K. M.; PICININ, M. E.; DEL SANTO, V. R.; SOUZA, G. B.; PEREIRA, C.A.M. Centesimal and mineral composition of powdered carob and its use on preparation and cceptability of an ice cream. Alimentos e Nutrição, Araraquara, v. 22 , n. 1, p. 129-136, jan./mar, 2011.

TACO. Tabela Brasileira de Composição de Alimentos. 4. ed. Campinas: UNICAMP/ NEPA, 2011. p. 161.

VORAGEN, A. G. J. Technological aspects of functional food-related carbohydrates. Trends in Food Science \& Technology. v. 9, n.. 8, p. 328-335, 1998.

YOUSIF, A. K., ALGHZAWI, H. M. Processing and characterization of carob powder. Food Chemistry, vol. 69, no. 3, p. 283-287, 2000. 